\title{
Strength-duration curve: a measure for assessing sensory deficit in peripheral neuropathy
}

\author{
WG FRIEDLI, M MEYER \\ From the Department of Neurology, University Hospital, Zürich, Switzerland
}

SUMMARY By using an isolated constant current stimulator producing true square-wave pulses, sensory strength-duration curves were obtained at various sites by percutaneous electrical stimulation. Strength-duration curves derived from normal groups were compared to those of patients with peripheral neuropathy. Stimulus strength at sensory threshold was shown to be a reproducible measure of sensory deficit, increasing parallel to the degree of axonal failure found by conventional methods. This may be useful as a complementary method in assessing peripheral neuropathy.

The fact that both muscle and peripheral nerve characteristically respond to electrical stimulation allows a number of electrodiagnostic procedures. The relationship between the strength of a stimulus and its duration for producing minimal excitation is expressed by the strenth-duration (S-D) curve. ${ }^{1}$ The standard type of S-D curve utilising visible muscle contraction has been applied to traumatic peripheral nerve injuries and lower motor neuron lesions. ${ }^{2-6}$ While electromyography provides extensive information as to the state of innervation of skeletal muscles, sensory conduction velocity often does not mirror the clinical state of the patient. For several reasons the quantitative examination of cutaneous sensation encounters serious problems. ${ }^{7}$ Both afferent and efferent fibres have been studied by various electrodiagnostic techniques: nerve excitability has been determined by the strength of the true square-wave stimuli required to elicit a barely visible nerve action potential at a recording site proximal to the stimulus. ${ }^{8-11}$ Stimulus strength at sensory threshold has been shown to be more reproducible than the one just evoking a sensory action potential. ${ }^{12}$ Sensory S-D curves obtained with behavioural psychophysical techniques have matched those obtained by electrophysiologic procedures. ${ }^{13}$ However, few clinicians have used cutaneous electrical stimulation for assessing peripheral nerve function ${ }^{14}$ and clinical feasibility has not been apparent in the past. According to our findings the sensory S-D

Address for reprint requests: Walter G Friedli, Neurologische Universitatsklinik Kantonsspital, CH-4031 Basel, Switzerland.

Received 23 June 1983. Accepted 2 August 1983 curve offers, to a certain extent, a useful method for assessing peripheral neuropathies.

\section{Methods}

Silver surface electrodes of $8 \mathrm{~mm}$ diameter filled with electrode cream were taped about $2.5 \mathrm{~cm}$ apart over the skin areas of interest. Skin preparation consisted of shaving the skin if necessary and cleaning it with ether and alcohol. The distal electrode was connected to the cathode of an isolated stimulation system consisting of an impulse generator (W-P Instruments Inc.) and a constant current stimulator (DISA $15 \mathrm{E} \mathrm{07).} \mathrm{The} \mathrm{latter} \mathrm{was} \mathrm{modified} \mathrm{for}$ external pulse width control and for extended pulse width of $50 \mathrm{~ms}$ (with approximately $10 \%$ pulse drop for $50 \mathrm{~ms}$ pulse duration). The amount of current in milliamperes required to produce a minimally perceptible sensation was determined for various durations of square-wave pulses ranging from 0.05 to $50 \mathrm{~ms}(0.05,0.06,0.08,0.10,0.20$, $0 \cdot 50,1 \cdot 0,2 \cdot 0,5 \cdot 0,10 \cdot 0,20 \cdot 0,50 \cdot 0 \mathrm{~ms})$. For each of the 12 pulse durations detection thresholds were obtained by a method of descending limits, continuously decreasing from a level at which the subject had a minimal sensation. Electrical stimuli were given at a rate of $0.5 / \mathrm{s}$ and were displayed on an oscilloscope screen in front of the subject. Usually three independent measurements were performed on each subject for each pulse duration tested, the series starting with $50 \mathrm{~ms}$ stimuli. S-D curves were obtained by plotting current intensities producing a just perceptible sensation against stimulus duration.

For each stimulation site studies were performed on a control group and a patient group. Each control group consisted of 10 to 12 healthy subjects without history or clinical evidence of neuromuscular disease. To ascertain the reproducibility of normal curves the same subject was tested in 10 independent series of measurements on successive days. During the measurements skin temperature was controlled by means of a digital thermometer and kept constant by an infrared lamp within the range of $1^{\circ} \mathrm{C}$. 
Results

S-D curves were surprisingly reproducible in healthy subjects on successive trials. The task was more difficult for some of the patients depending on the degree of sensory impairment, especially if paraesthesiae were present. The subjects compared the sensation produced by electrical stimuli to the sensation of being tapped, that is, a stimulus tactile in quality.

In a first series of experiments normal S-D curves were obtained at various stimulation sites. In one group of 12 healthy subjects sensory thresholds were measured bilaterally in the cutaneous field of the superficial peroneal nerve. The electrodes were placed on the proximal dorsum of the foot following the tendon of extensor hallucis longus. Average thresholds across trials and different subjects are presented separately for the two sides of the body in fig $1 \mathrm{~A}$. A rapid decrease of the threshold current with increasing stimulus duration up to 0.5 to $1.0 \mathrm{~ms}$ was observed while there was little change of threshold current with longer stimuli. The minimal current required to produce cutaneous sensation also with very long current (rheobase) was achieved by pulse durations of approximately $10 \mathrm{~ms}$. Sensory thresholds were found to be lower for the left compared to the right side of right-handed people in both upper and lower extremities. Although the difference in absolute values may be disregarded, this asymmetry was a consistent finding in our normal subjects.

Reproducibility of S-D curves for the same stimu-

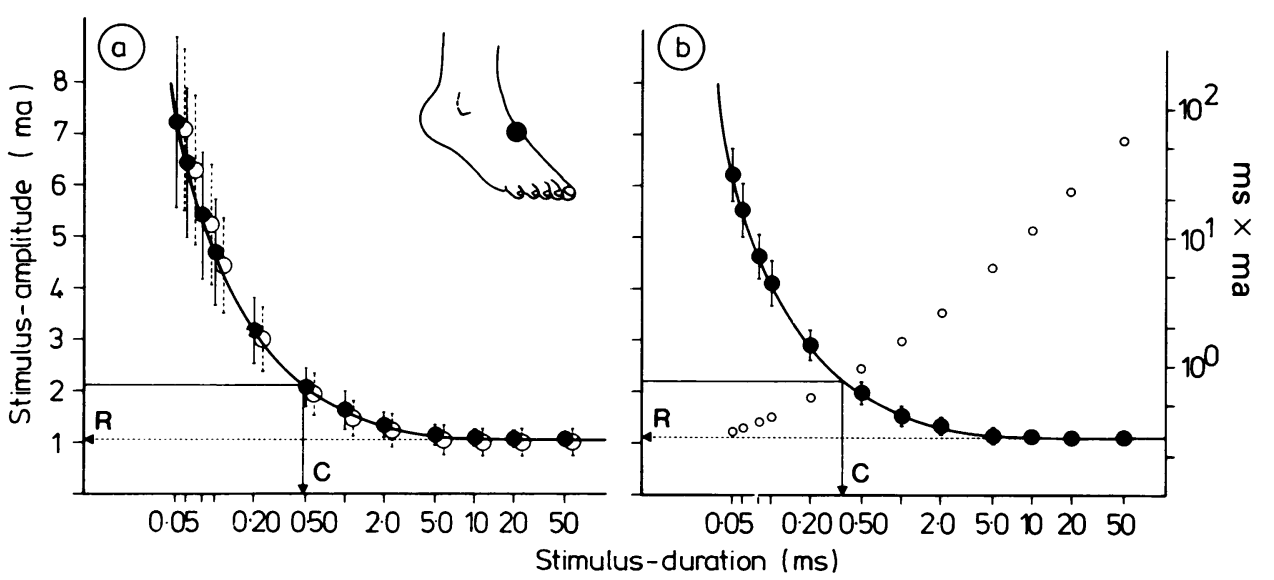

Fig 1 Strength-duration curve for rectangular current pulses on the dorsum of the right (O) and left (O) foot, ie in the cutaneous field of the superficial peroneal nerve. Rheobase $(R)$ and chronaxy $(C)$. $(A)$ Average of 12 healthy subjects ranging in age from 25 to 41 years. (B) Reproducibility of $S$-D curve for 1 subject of 27 years, same stimulation site. Average of 10 series of independent measurements performed on successive days. Energy necessary for minimal sensation as a function of pulse duration (O in $B$ ), showing temporal summation properties.

lation site is shown in fig $1 \mathrm{~B}$ for the same subject. While being in the same range, the threshold values showed smaller standard deviations when compared with the whole group, even if independent measurements were performed on different days. A similar degree of reproducibility was observed in individual subjects for the cutaneous field of the median or ulnar nerve.

Temporal summation properties are represented by the product of current strength and duration at threshold levels (stimulus energy in microcoulombs) as a function of stimulus duration (fig 1B). According to the Bunsen-Roscoe law or Bloch's law stimulus energy at threshold levels remains constant for stimulus durations less than the "critical duration" specific for a sensory modality. For vision, reciprocity between luminance and time at threshold has been found to be limited to durations of about $10 \mathrm{~ms}$ in the paramacular region ${ }^{15}$ while the critical duration was found to be considerably longer for other sensory modalities. In agreement with Rollman's findings our data suggest a very small critical duration of less than $0.10 \mathrm{~ms}$ for cutaneous electrical stimulation. The absence of a total temporal summation in our graphs was probably due to the fact that minimum pulse duration was limited to $0.05 \mathrm{~ms}$. Different degrees of partial temporal summation were found for successive periods, the first extending to about $0.10 \mathrm{~ms}$, and a second to about $1.0 \mathrm{~ms}$. There was no temporal summation for pulse durations beyond $10 \mathrm{~ms}$ and the function had a slope of 1.0 .

To allow comparison between different stimula- 


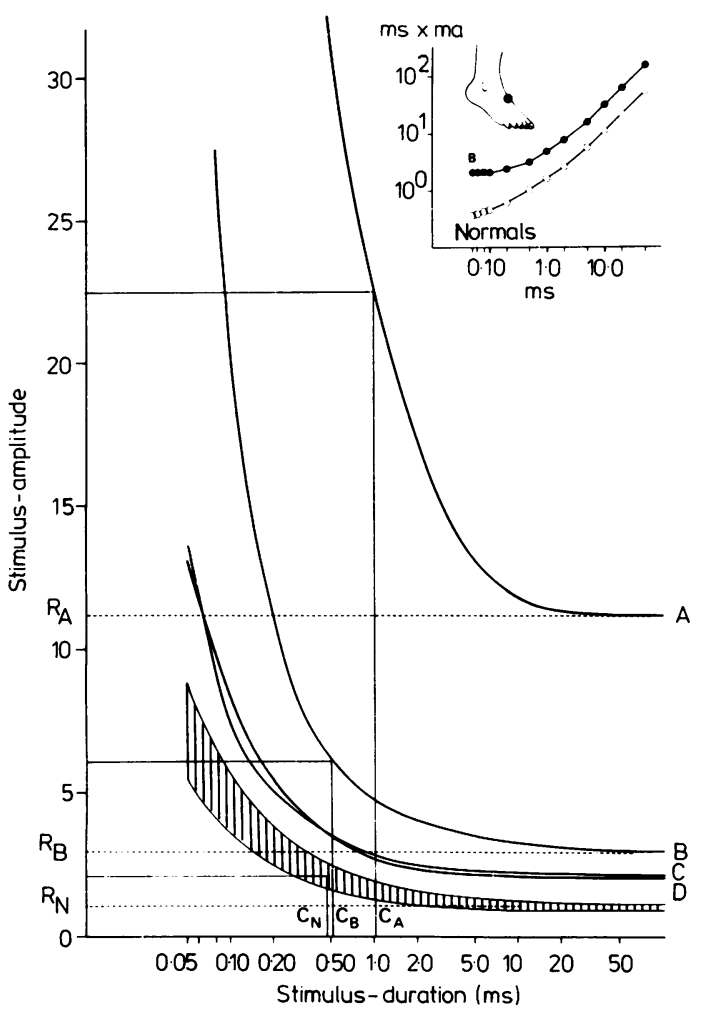

Fig $2 S$-D curves (superficial peroneal nerve) in neuropathic disease compard to normals of fig 1 (hatched area: mean \pm 1 standard deviation). Individual curves from patients with angiopathic neuropathy $(A)$, alcoholic $(B)$, uraemic $(C)$ and diabetic $(D)$ polyneuropathy (details in table). Compare marked differences of rheobases and the similar chronaxy values for the different conditions. Upper graph: threshold energy as a function of pulse duration for patient $B$ and healthy group showing differences in temporal integration properties between the two.

tion sites on the same subject, threshold measurements were performed by stimulating in the cutaneous fields of the sural, superficial peroneal as well as lateral femoral cutaneous nerve. Compared with the area of the superficial peroneal nerve lower thresholds were found on the external border of the foot and even lower values were found on the lateral aspect of the thigh in this group of healthy subjects. For all subjects there was a consistent rank of absolute thresholds according to the stimulation site; the values of the whole group were not significantly different ( $p<0.5$ to $p<0.05$ for short stimuli up to $0 \cdot 10 \mathrm{~ms})$.

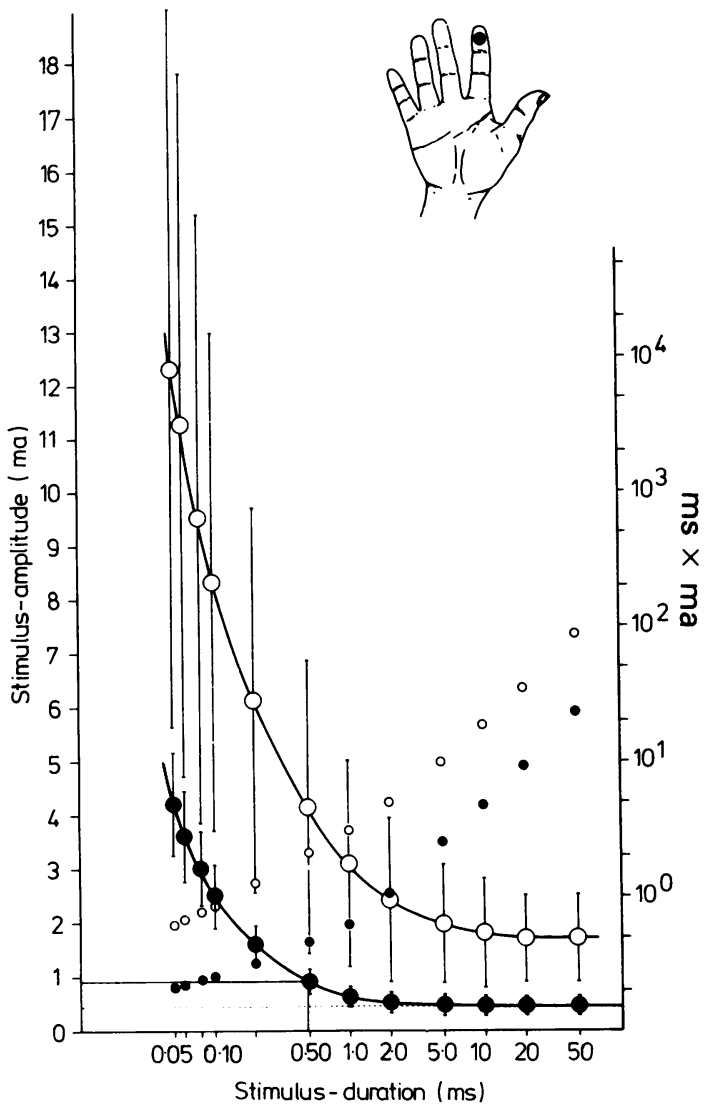

Fig $3 S$-D curves for the median nerve (stimulating cathode on the index finger). Average of 8 healthy subjects from 26 to 41 years $(\bullet)$ for both hands $(n=16)$. Note the difference of absolute threshold values between median and superficial peroneal nerve (fig 1). Mean and standard deviations of 6 patients $(O)$ with severe carpal tunnel syndrome (sensory action potentials of small amplitude markedly delayed, if recordable at all). Comparison of the two threshold energy functions reveals differences in absolute values and temporal integration properties.

Sensory S-D curves were obtained from 25 patients with various kinds of peripheral neuropathy. Some examples are given for stimulation in the cutaneous field of the superficial peroneal nerve in fig 2 (see table for case descriptions). S-D curves of the pathological subject groups are compared with the group of normal subjects in fig 1 . The rate of climb in the pathological curves was larger from right to left compared to that of the normal curves. However, even if present, this variation of slope was too small to be of any practical relevance. In general, the sensitivity threshold in terms of stimulus current was raised over the whole range of 
Table Case descriptions (patients of figure 2). Rating of pathological conditions: 0 normal, 1 mild, 2 moderate, 3 severe

\begin{tabular}{|c|c|c|c|c|c|c|c|c|c|c|c|c|c|c|}
\hline 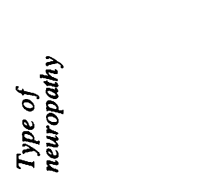 & $\stackrel{\infty}{\infty}$ & らั้ & 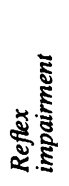 & 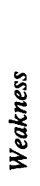 & $\frac{\pi}{5}$ & 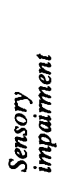 & 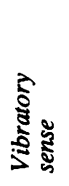 & స & 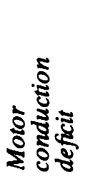 & 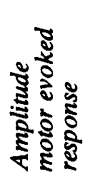 & 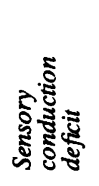 & 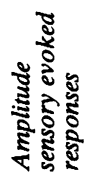 & 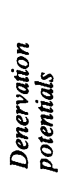 & 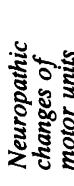 \\
\hline $\begin{array}{l}\text { A } \\
\text { Wegener's } \\
\text { granulomatosis }\end{array}$ & 55 & $\mathbf{M}$ & 3 & 2 & 3 & 2 & 3 & $\begin{array}{l}\text { painful } \\
\text { dysaesthesia } \\
\text { on both } \\
\text { feet }\end{array}$ & 1 & 1 & 2 & 2 & 2 & 2 \\
\hline B Alcoholic & 33 & $\mathbf{F}$ & 3 & 3 & 3 & 1 & 2 & $\begin{array}{l}\text { bilateral } \\
\text { foot drop, } \\
\text { unable to } \\
\text { walk }\end{array}$ & 1 & 1 & 2 & 2 & 3 & 3 \\
\hline $\begin{array}{l}\text { C } \\
\text { Uraemic (chronic } \\
\text { renal failure) }\end{array}$ & 35 & $\mathbf{M}$ & 2 & 1 & 1 & 1 & 2 & & 3 & 2 & 2 & 1 & 0 & 1 \\
\hline $\begin{array}{l}\text { D } \\
\text { Diabetic }\end{array}$ & 66 & $\mathbf{M}$ & 2 & 1 & 0 & 2 & 2 & $\begin{array}{l}\text { bilateral } \\
\text { entrapment } \\
\text { neuropathies } \\
\text { of ulnar and } \\
\text { median nerve }\end{array}$ & 2 & 2 & 1 & 1 & 0 & 1 \\
\hline
\end{tabular}

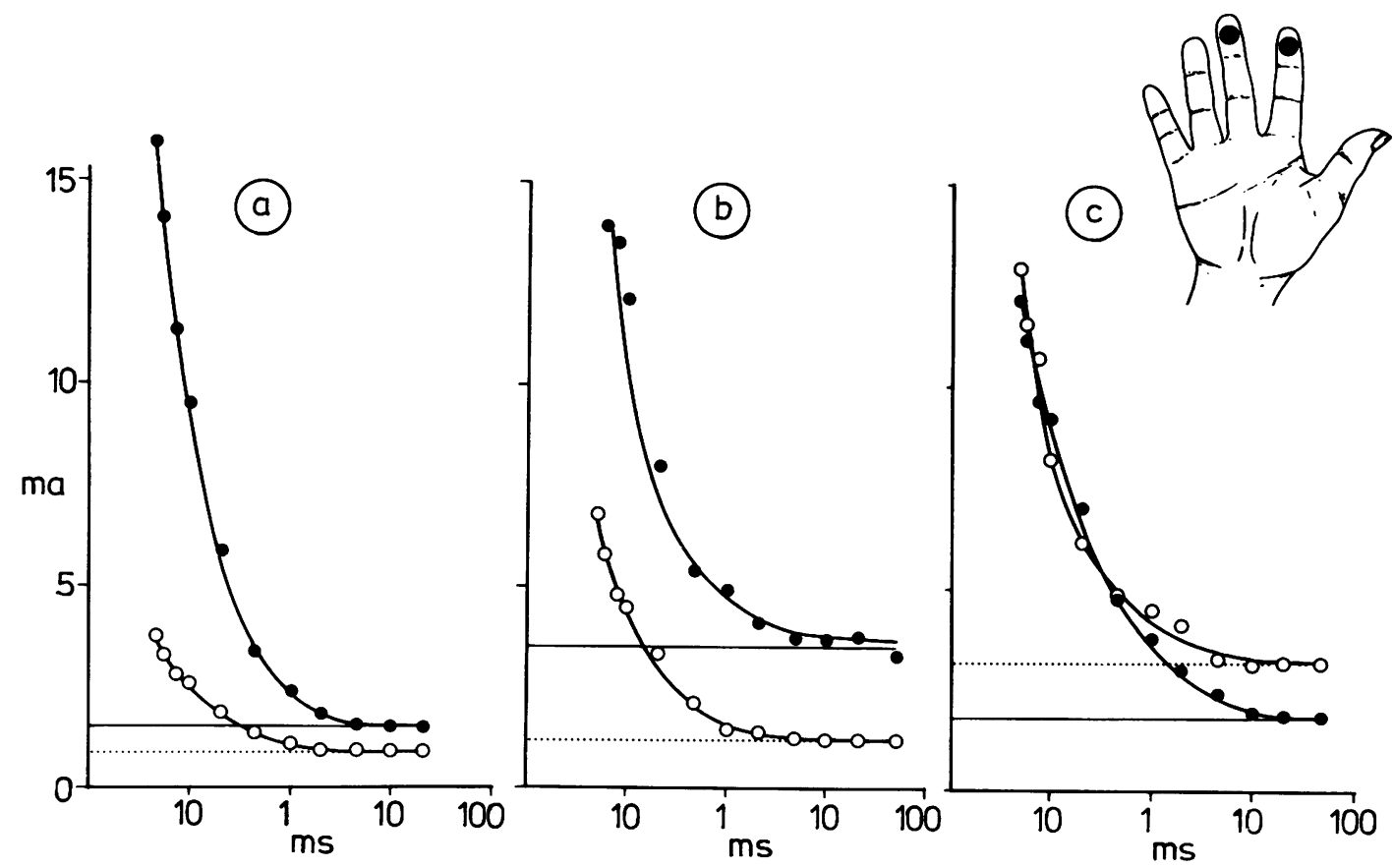

Fig 4 (A) 41-year-old male. Traumatic partial lesion of the right median nerve in the distal forearm 4 months before examination. Severe sensory impairment to all modalities in the index (O), markedly less in the middle finger (O). No sensory action potentials from digit to wrist. Note the difference between $S$ - $D$ curves on the two fingers which is in accordance with the clinical findings. (B) 24-year-old female, suffering from symptoms of a moderate carpal tunnel syndrome (sensory conduction of $39 \mathrm{~m} / \mathrm{s}$, small sensory action potential of $4 \mu V$ ). $S-D$ curve on the right index finger before $(O)$ and after (O) paraneural infiltration with an anaesthetic into the carpal tunnel (when no action potential could be recorded any more). (C) $S$-D curves (index finger) of a 60-year-old male patient with severe carpal tunnel syndrome before $(O)$ and 3 months after $(O)$ surgical decompression of the right median nerve. Note the difference of rheobase before (no sensory action potential, distal motor latency of $6.3 \mathrm{~ms}$ ) and after surgery (markedly delayed action potential of $2 \cdot 5 \mu \mathrm{V}$, motor latency of $5 \cdot 1 \mathrm{~ms}$ ). 
pulse duration as is shown by the curve shifting upward and to the right. This shift was also found for stimulus energy at threshold levels (fig 2, upper graph): the patient curve shows an overall rise of threshold energy. A comparison of the slopes of the two energy functions revealed changes in temporal summation properties. In contrast to healthy subjects, a reciprocity between stimulus strength and time at threshold levels was found for pulse durations up to $0.10 \mathrm{~ms}$ in subjects with severe neuropathy. Hence, not only partial temporal summation was extended to longer pulse durations but also the critical duration was prolonged in the pathological case.

S-D curves for the median and ulnar nerve were obtained by stimulating the distal phalanges of the index and middle finger or of the little finger respectively. Normal curves were compared with those found with various nerve lesions. Even if there was clear clinical and electroneurographic evidence of entrapment neuropathy, sensitivity thresholds often did not reflect the pathological condition. However, S-D curves were significantly altered with a severe carpal tunnel syndrome, characterised by marked conduction deficits, electromyographic abnormalities in the thenar muscles or loss of the sensory action potential (fig 3). In these cases, serial S-D curves after surgical decompression of the median nerve displayed a functional improvement (fig 4C). There was also a clear relationship between sensory deficits due to traumatic nerve lesions and the behaviour of sensitivity thresholds as a function of stimulus duration (fig 4A).

\section{Discussion}

Together with the clinical and electrodiagnostic evaluation of a peripheral neuropathy, the systematic examination of sensory strength-duration curves provides useful help in diagnosis.

The characteristics of psychometric functions for electrocutaneous stimuli have suggested that current pulses bypass the receptors and directly excite sensory fibres ${ }^{1617}$ as supported by other results. ${ }^{18-20}$ Moreover, temporal integration properties of psychophysical S-D curves were consistent with stimulation of large A-fibres by electrocutaneous pulses. ${ }^{16}$ According to previous findings fibres with large diameter have faster velocities and lower thresholds than fibres with small diameter..$^{21-23}$

The correlation of the S-D curve with pathological clinical and electrodiagnostic findings in the same patient provides further information as to the anatomical substrate tested by the psychophysical technique. Both patients A and B of fig 2 had a symmetrical motor and sensory polyneuropathy with subnormal to normal nerve conduction and the electromyographic equivalents of a severe axonal lesion. On the other hand, the findings in patients $\mathrm{C}$ and $\mathrm{D}$ are characterised by motor and sensory conduction defects with little evidence of denervation. This is in agreement with the fact that S-D curves are hardly impaired in chronic entrapment neuropathies (for example, the median nerve in fig 4B) but affected in proportion to the incidence of axonal lesion (for example, patients of fig 3 ).

Discontinuities in the classical S-D curve result from the difference in excitability threshold between muscle and nerve tissue. Therefore, sensory S-D curves representing properties of afferent nerve fibres are expected to be smooth also in case of partial denervation. Pathological conditions are characterised by an over-all increase in threshold excitability providing both a shift of the curve and changes in temporal integration properties. There are various possible explanations for this behaviour; it may be due to a threshold increase of the A fibres, or stronger stimuli may be required to recruit an increasing number of $\mathbf{A}$ fibres for minimal sensation. Another possibility is that stronger pulses may provide excitation of other types of afferent fibres in addition to A fibres. This is suggested by the changes in temporal summation properties. However, our results cannot give additional information about the elements of sensory S-D curves.

Assessment of sensory impairment is part of the clinical evaluation of peripheral neuropathies. For several reasons quantification of cutaneous sensation by clinical examination is inaccurate and calls for methods of measurement using "adequate" stimuli. ${ }^{24-26}$ Both sensory S-D curves and quantitative sensory tests are complementary to the clinical evaluation of peripheral nerve disorders. The fact that the sensory threshold is mostly affected with axonal lesions suggests a valuable complement to conventional electrodiagnostic techniques.

The simplicity of the method (no complex recording and averaging equipment) represents a further advantage in comparison with electroneurographic procedures. However, the isolated constant-current stimulator has to be capable of producing true square-wave stimuli over a wide range of pulse durations. Moreover, the subject tested must be attentive and cooperative during the behavioural assessment of peripheral nerve function. The examination can be reduced to a few representative pulse-durations. The amount of current of infinite duration required to produce minimal sensation (rheobase) represents a valuable measure and approximates stimulus durations of 10 to $50 \mathrm{~ms}$. The chronaxy, that is the duration of a threshold stimulus of twice the rheobase, proved to be less informative, expecially because it 
is based on calculation. Temporal integration properties and critical duration are useful parameters but require several measurements in the low range of stimulus duration. For each laboratory S-D curves must be based on normal values (specific for age and stimulation site). However, bilateral comparison within the same subject might be indicative of mononeuropathy.

The authors thank Dr Dorothea Weniger for her help in preparing this manuscript and $\mathrm{Mr}$ Joseph Müller for photographic work.

\section{References}

${ }^{1}$ Wynn Parry CB. Strength-duration curves. In: Licht S, ed. Electrodiagnosis and Electromyography. 3rd ed. New Haven: Elizabeth Licht, 1971:241-71.

${ }^{2}$ Adrian ED. The electrical reactions of muscles before and after nerve injury. Brain 1916;39:1-33.

${ }^{3}$ Pollock LJ, Golseth JG, Arieff AJ. Strength-frequency curves in electrodiagnosis of experimentally produced peripheral nerve lesions. Surg Gynecol Obst 1945;80:235-42.

${ }^{4}$ Newman HW, Livingstone WK. Electrical aids in prognosis of nerve injuries. J Neurol Neurosurg Psychiatry 1947;10:118-21.

s MacKenzie IG. Electrical reactions of muscle in poliomyelitis. Proc $R$ Soc Med 1949;42:488-90.

6 Wynn Parry CB. Electrical methods in diagnosis and prognosis of peripheral nerve injuries and poliomyelitis. Brain 1953;76:229-65.

${ }^{7}$ Dyck PJ. Quantitation of cutaneous sensation in man (chap 22). In: Dyck PJ, Thomas PK, Lambert EH, eds. Peripheral Neuropathy Vol. 1. Philadelphia: Saunders, 1975:465-79.

${ }^{8}$ Heckmann JR. Excitability curve: a new technique for assessing human peripheral nerve excitability in vivo. Neurology (Minneap) 1972;22:224-30.

9 Powers RE, Swick HM, McQuillen MP. Human nerve excitability. J Neurol Neurosurg Psychiatry 1978; 41:642-8.

${ }^{10}$ Veale JL, Mark RF, Rees S. Differential sensitivity of motor and sensory fibres in human ulnar nerve. $J$ Neurol Neurosurg Psychiatry 1973;36:75-86.

$"$ Wright EA, McQuillen MP. Hypoexcitability of ulnar nerve in patients with normal motor nerve conduction velocities. Neurology (Minneap) 1973;23:78-83.

12 Buchthal F, Rosenfalck A. Evoked action potentials and conduction velocity in human sensory nerves. Brain Res 1966;3:1-122.

${ }^{13}$ Rollman GB. Behavioral assessment of peripheral nerve function. Neurology (Minneap) 1975;25:339-42.

${ }^{14}$ Bourguignon G. La Chronaxie chez l'Homme. Etude de Physiologie Générale (normale et pathologique) des Systemes Neuro-musculaires et Sensitifs. Paris: Masson, 1923.

${ }^{15}$ Battersby WS, Schuckman H. The time course of temporal summation. Vision Res 1970;10:263-72.

${ }^{16}$ Rollman GB. Electrocutaneous stimulation: Psychometric functions and temporal integration. Percept Psychophys 1969;5:289-93.

17 Rollman GB. Detection models: Experimental tests with electrocutaneous stimuli. Percept Psychophys 1969; 5:377-80.

${ }^{18}$ Gildemeister M. Untersuchungen über die Wirkung der Mittelfrequenzströme auf den Menschen. Pfügers Arch Ges Physiol 1944;247:366-404.

${ }^{19}$ Higgins JD, Tursky B, Schwartz GE. Shock-elicited pain and its reduction by concurrent tactile stimulation. Science 1971;172:866-7.

${ }^{20}$ Hawkes GR. Cutaneous discrimination of electrical intensity. Am J Psychol 1961;74:45-53.

${ }^{21}$ Blair EA, Erlanger J. A comparison of the characteristics of axons through their individual electrical responses. Am J Physiol 1933;106:524-64.

${ }^{22}$ Gasser HS, Grundfest H. Axon diameters in relation to the spike dimensions and the conduction velocity in mammalian A fibres. Am J Physiol 1939;127:393414.

${ }^{23}$ Wiederholt WC. Stimulus intensity and site of excitation in human median nerve sensory fibres. $J$ Neurol Neurosurg Psychiatry 1970;33:438-41.

${ }^{24}$ Dyck PJ, Schultz PW, O'Brien PC. Quantitation of touch-pressure sensation. Arch Neurol 1972;26: 465-73.

${ }^{25}$ Fruhstorfer H, Lindblom U, Schmidt WG. Method for quantitative estimation of thermal thresholds in patients. J Neurol Neurosurg Psychiatry 1976;39: 1071-5.

${ }^{26}$ Lindblom U. Quantitative testing of sensibility including pain (chap 8). In: Stalberg E, Young RR, eds. Neurology, vol 1: Clinical neurophysiology. London: Butterworth, 1981:167-90. 